استخدام الثبكات العصبية في التكهن بالسلسلة الزمنية لاستهلاك الطاقة الكهربائية في مدينة الموصل

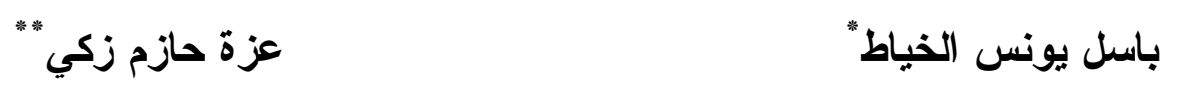

(المستخلص

يُعَدّ التكهن بالسلوك المستقبلي للسلاسل الزمنية من الموضوعات المهمة في العلوم الإحصائية، وذلك للحاجة إلبه في مجالات الحياة جميعاً، وقد نز ايد الاهتمام بموضوع التكهن خلال السنو ات الأخيرة وظهرت أساليب حديثة خاصة في مجــال

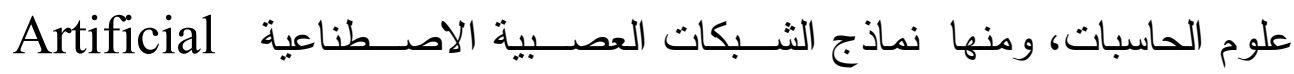
Neural Networks نموذج، ولا تحتاج إلى افتر اضـات لطبيعة السلسلة الزمنية. بالمقابل فــان طر ائــق

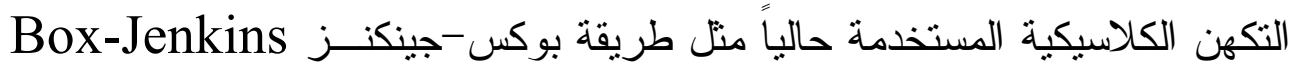
قد يصعب فيها تشخيص السلسلة ونمذجتها لأنها تفترض شروط صـــارمة. لــذلك ظهرت الحاجة الى مقارنة الطرائق الكلاسيكية المستخدمة في الــتكهن بالسلاسـلـ الزمنية مع أسلوب الثبكات العصبية لإيجاد الأسلوب الأكثر كفاءة في التكهن.

\title{
The Use of Neural Networks in Forecasting of Time Series with Application on Consumption of Electrical Power in Mosul City
}

\section{ABSTRACT}

Forecasting of future behavior of time series is one of the important subjects in statistical sciences, because of its important need in different areas of life. Interest in forecasting had increased in the recent years, and some new techniques in the

$$
\begin{aligned}
& \text { " " استاذ/كلية علوم الحاسبات و الرياضيات/جامعة الموصل }
\end{aligned}
$$

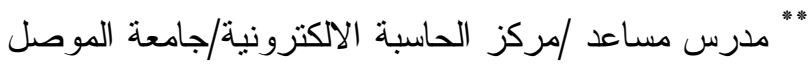

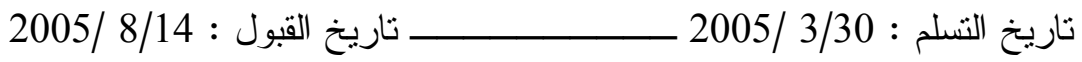


field of computer science, like Artificial Neural Networks (ANN), had appeared. These techniques have the ability learning and self- adaptation with any model, and don't need assumptions on the nature of time series. On the other hand the classical forecasting methods, like Box-Jenkins method, need hard conditions. Hence, the need for a comparison between classical methods in forecasting of time series with ANN technique arose.

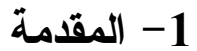

منذ مطلع العقد السابع من القرن العشرين ظهر اهتـــام متز ايـــــــــــلـ السلاسل الزمنية وطر ائق التكهن بقيمها المستقبلية، وفي بداية الثمانينات من القرن

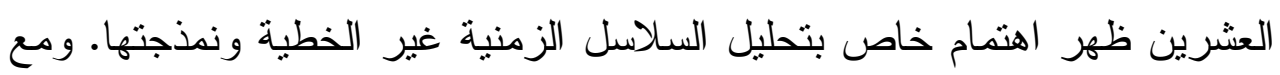

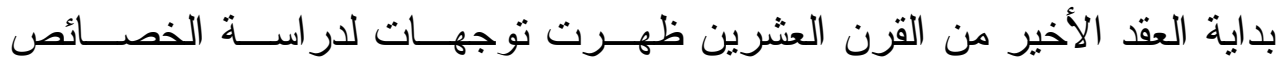

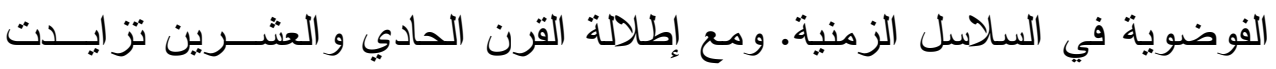
الاهتمامات في دراسة السلاسل الزمنية، وخاصة من خلال علاقتها الوثيقة بالنظم

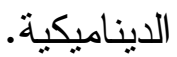

يرتكز تحليل السلاسل الزمنية على ثلاث ركائز تتمنل بثلاثة افتر اضات رئيسة:

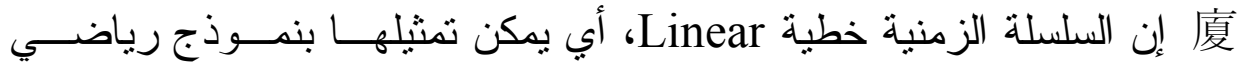
廈 廈

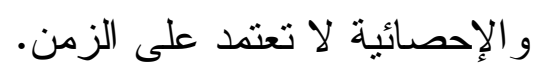

ان الاففراض الأول (الخطية) قد تم تجاوزه في العقد الثامن مــن القــرن

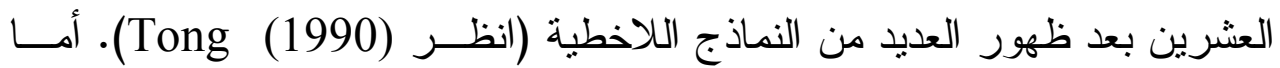

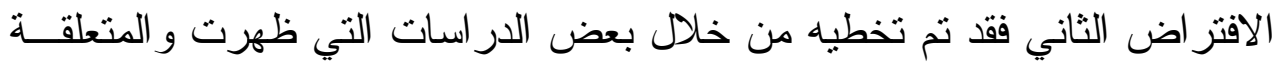

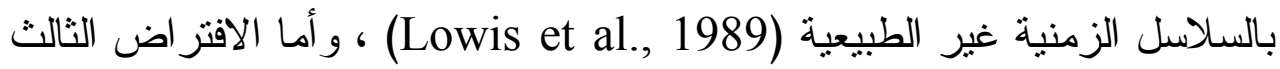

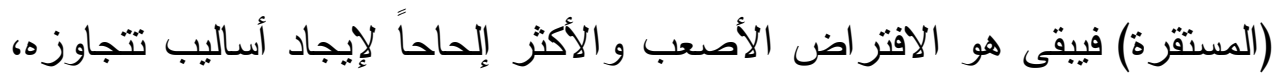
وذللك لان اغلب السلاسل الزمنية الو اقعية هي سلاسل غير مستقرة. 
من الأهداف الأساسية للعلوم الإحصائية التقدير و التكهن بالسلوك المستقبلي

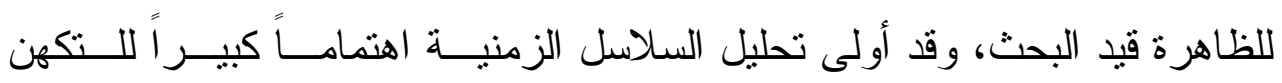
و اقترحت العديد من الأساليب لغرض إجر اء التكهن.

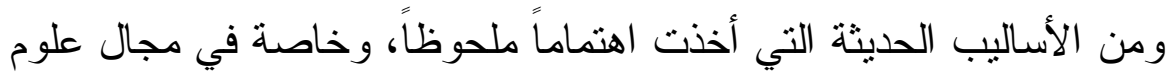

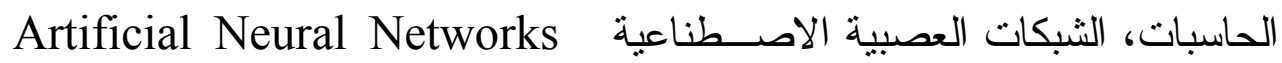
(ANNs) يحاكي النظام البيولوجي العصبي. إن المفتاح الأساسي لهذا النموذج هو بناء هيكل

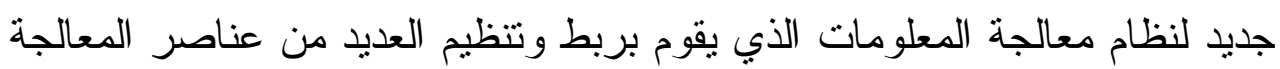
المرتبطة مع بعضها وهي (العصبونات) التي تعمل بشكل متتاسق لحل المشكلة قيد بلئ

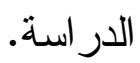

و الثبكات العصبية الاصطناعية (ANNs) تتعلم بطريقة تشابه تعلم الإنسان

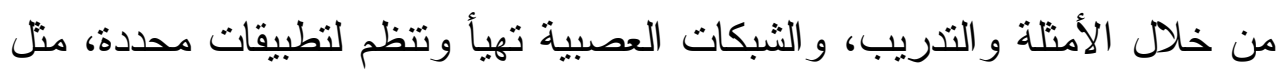

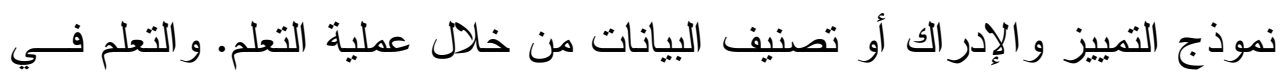
النظام البيولوجي يستخدم تكييف نقاط الاشتباك العصبي بين العصبونات، و وذه هي وني الفكرة الجوهرية في عمل الثبكات العصبية.

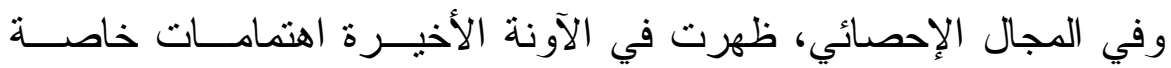

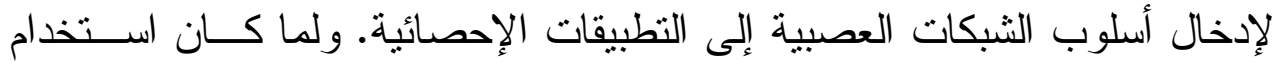
أسلوب الثبكات العصبية لا يتطلب افتز اضات عن طبيعة السلسلة الزمنية، كونها

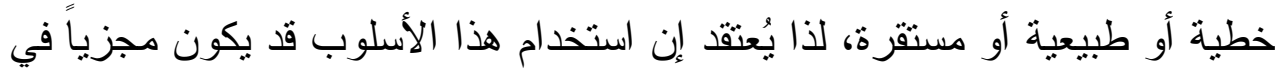

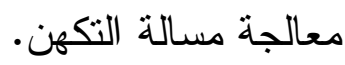

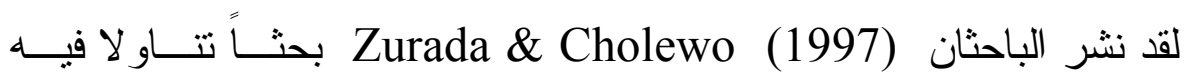

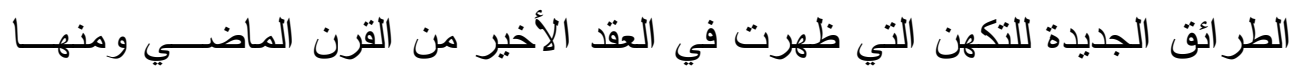
الثبكات العصبية الاصطناعية Artificial Neural Networks (ANNs) وقدما

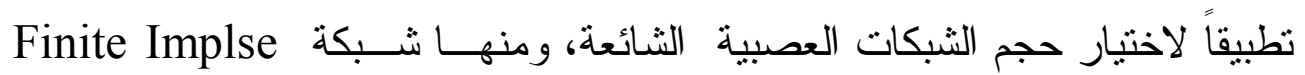
Elman Network Response Neural Network (FIR) و الثبكات المنطورة منها. و أجريا التكهن باستخدام التباين وتقدير الثرعية المتقاطع 
اللاخطي Nonlinear Cross Validation، ونوصلا إلى أن افضل شبكة للتكهن

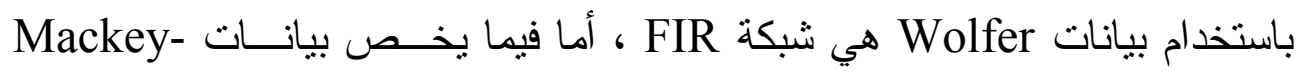
Atiya et الاقتصادية فقد كانت افضل شبكة هي Glass

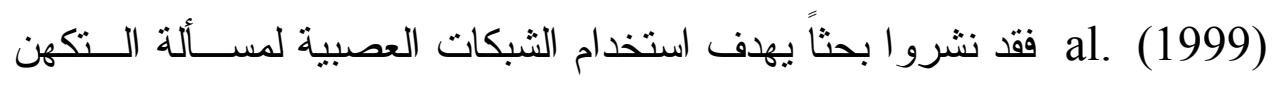

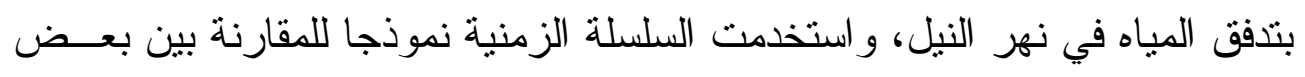

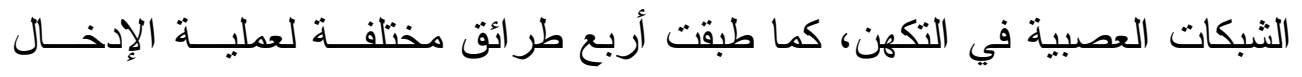

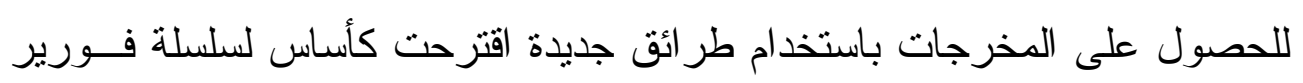
المتقطعة. وقدم Sansom \& Saha (1999) بحثاً للتكهن بأســعار الكهربــاء فـي

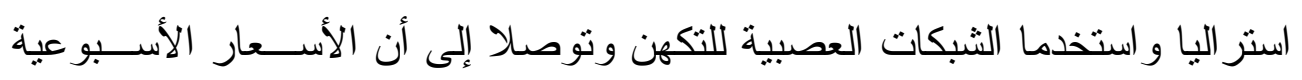
ثابتة ولكنها تتغير كل ستة انثهر • الثنا أما (2001) Lotric and Dobnikar فقد قدما بحثاً لتقليل حالة التشويش في التكهن للسلاسل الزمنية، وأُستخدمت شبكات عصبية منعددة الطبقات (noise)

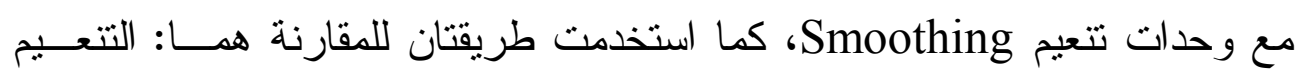

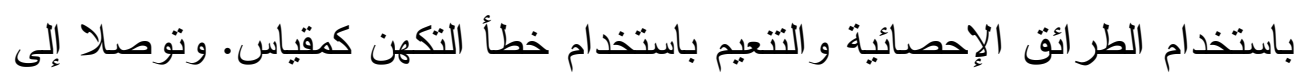

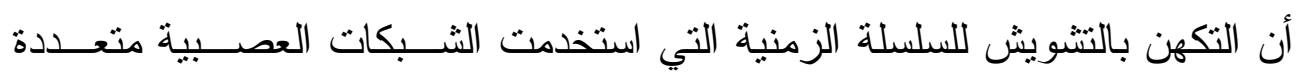

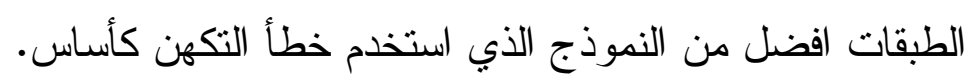

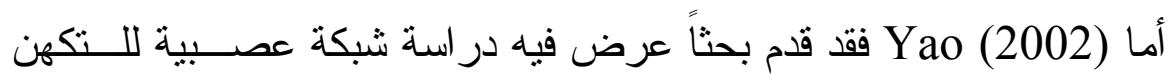

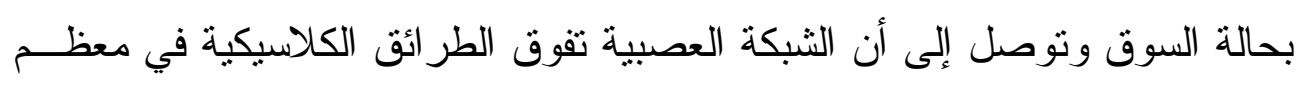

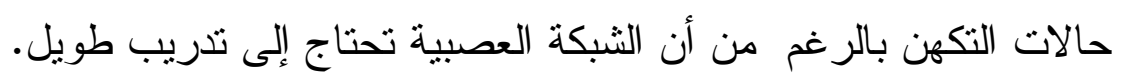

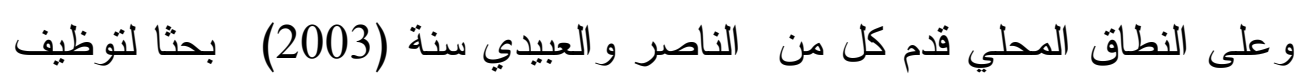

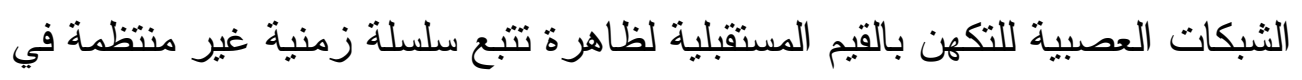

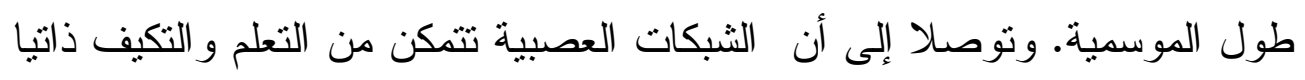

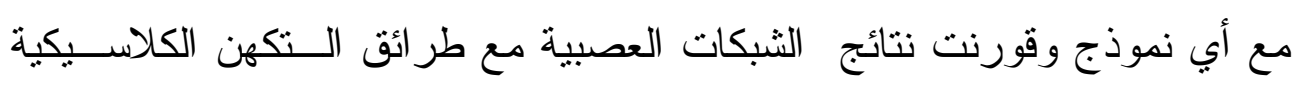
للتعرف على سلوكية الثبكات العصبية وجودنها. 
2- الثبكات العصبية الاصطناعية

تعني الثبكة العصبية في حقل تقنية المعلومات، نظاما من البرامج و هياكل

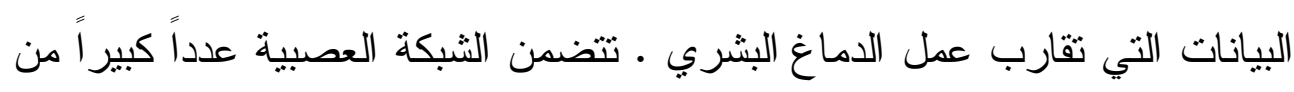

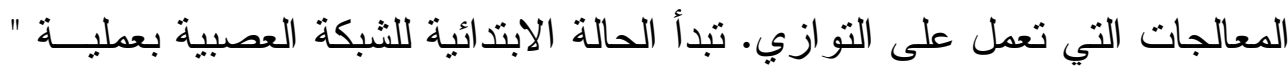

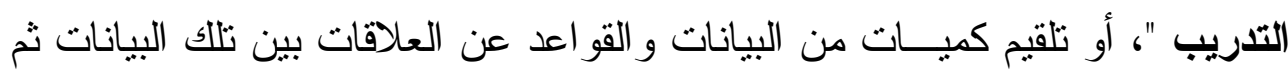

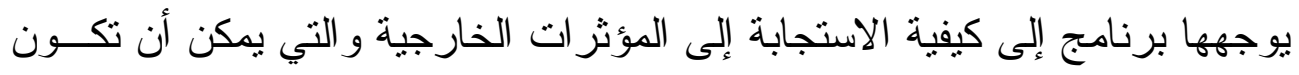

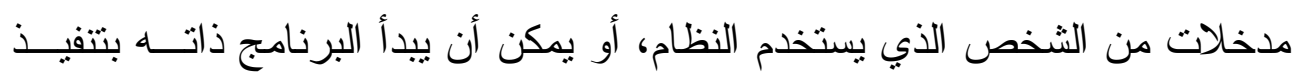
مهمة معينة. إن المفتاح لنموذج الثبكة العصبية الاصطناعية هو الهيكل لنظــام معــالج المعلومات الذي يقوم بربط وتتظيم عدد كبير من عناصر المعالجة المرتبطة داخليا

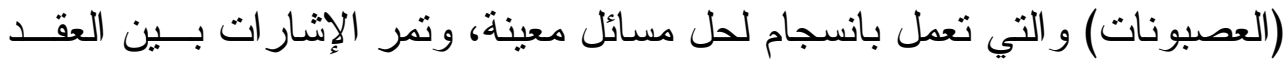

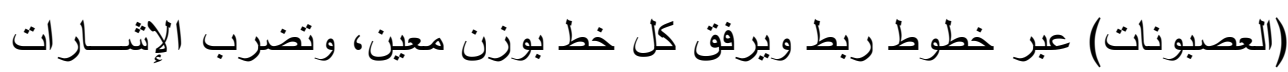

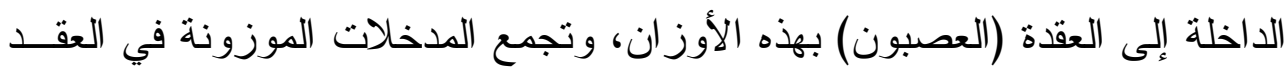
أو العصبونات وبعد ذلك تتم معالجة مخرجات كل عقدة بواسطة دالة غير خطيــة

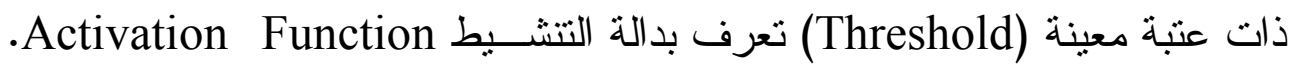
و الثكل (1) يوضح شبكة عصبية اصطناعية بسيطة.

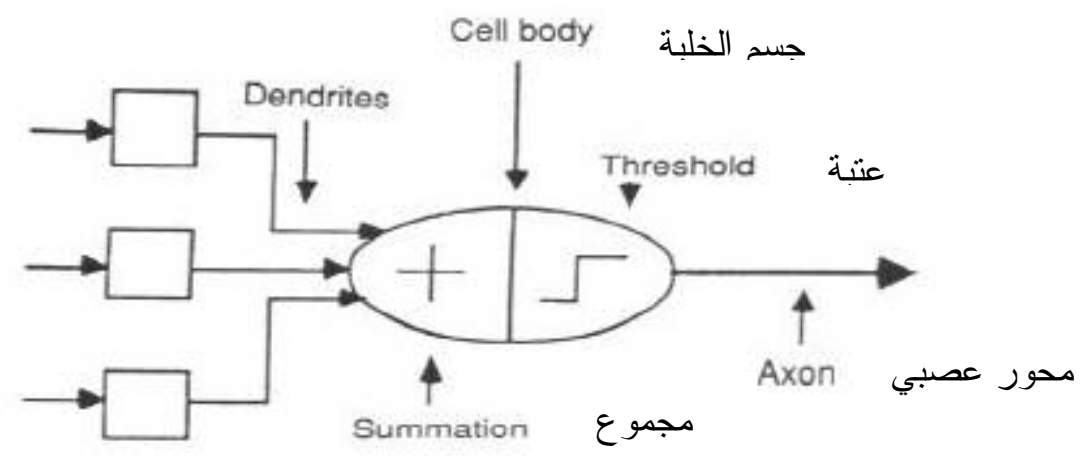

الثكل (1): جزء من الثبكة العصبية الاصطناعية. 
يمكن تعريف التعلم بأنه عملية ضبط الأوز ان لجعل الخلية تطلــق إثـــارة استجابة للأمتلة من مجموعة معينة. وكما هو معروف، فان الشبكة العصبية هـي مجموعة عصبونـات Neurons و اوز ان منظمة، و العصبونات منظمة في طبقــات، وكل عصبون في كل طبقة له وزن مرتبط مع كل عصبون فــي الطبقــة الاتيــة. و الثبكات العصبية الاصطناعية تتعلم عن طريق وسائل معينة تغير قــوة الــربط الداخلي، و هذا يدعى "تكييف الوزن" ويحدث خلال عملية تدعى "مرحلة التدريب". ومجمو عة الإدخال الخارجي تدعى "مجموعة التدريب للشبكة" أو (عينة التدريب)

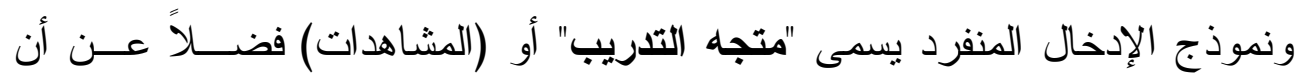
الثبكة يمكن أن تستقبل عن طريق التغذيــة العكسـية Feedback، أو بمكـن أن أن تستخدم التغذية العكسية مع معلومات إضافية لتحديد حجم التغير في الأوزان. 2-2 الثبكات العصبية ذوات الانتشار العكسي معية

\section{Backpropagation Neural Networks}

إن شبكات التغذية الخلفية أو العكسية يمكنها أن تمتلك إثار ات تتحرك أو تتتقل في كلا الاتجاهين، وهي شبكات حركية تتغير استقر اريتها باستمر ار إلى حين

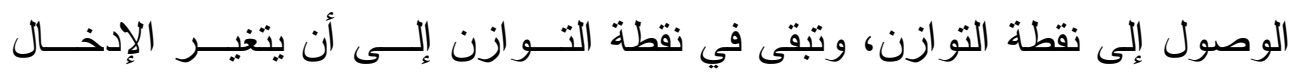

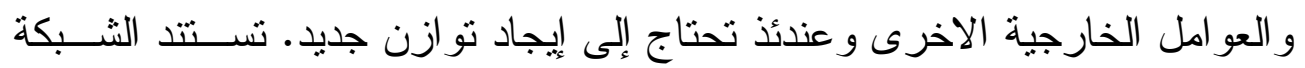

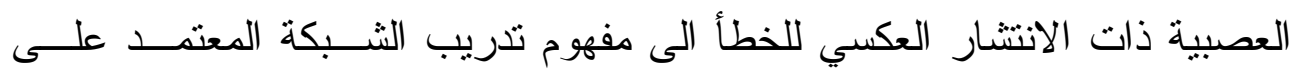
متوسط مربع الخطأ، وذللك بإيجاد القيمة الصغرى لمربــع الخطــأ الكـــي لقيمـــة المخرجات المحسوبة من قبل الشبكة اذ تحدّث الأوزان بين الطبقات لحين الوصول إلى الأوزان المثلى التي تحدث افضل توفيق للنموذج (الناصر و العبيدي، 2003).

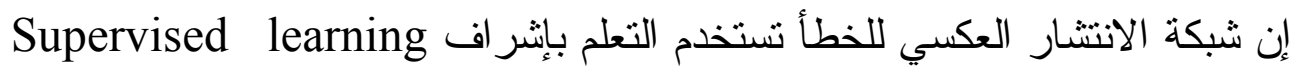

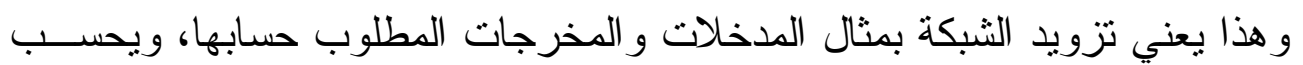
الخطأ (الفرق بين القيم الحقيقية و المتوقعة). وفكرة شبكة الانتشار العكسي هو تقليل الخطأ، إلى أن تتعلم الثبكة عن طريق التدريب، ويبدأ التدريب بأوزان عثـــوائية Gershenson, ) بهدف تكييف الثبكة إلى أن يصل الخطأ إلى اقـلـ مـــا يمكـنـ 


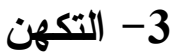

إن الفكرة الأساسية للتكهن بالسلسلة الزمنية هي إيجاد الصيغة الرياضــية

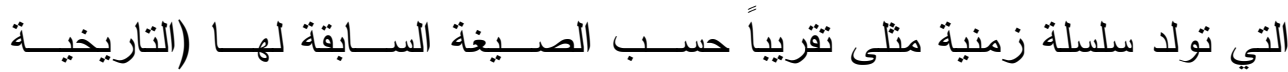

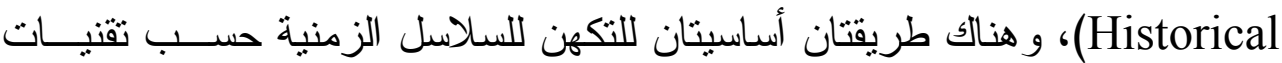
بوكس- جينكنز هما: الاسقاط الذاتي للسلســلة الزمنيــة ( Self-Projecting on (Time Series

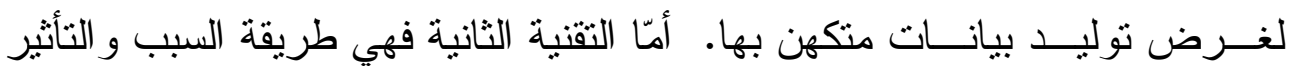

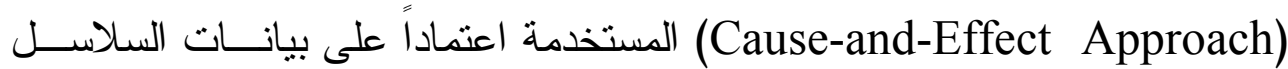

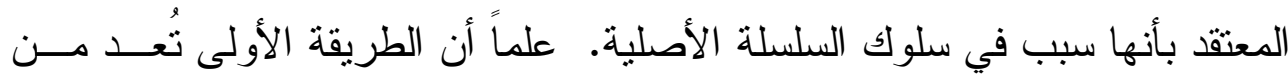
ابرز سمات طريقة التكهن لبوكس-جينكنز، اذ أن الهدف الأساسي لها هو إيجــــاد الهاديا صيغــة مناسـبة للتكهن بحيث تجعل البو اقي (Residuals) اقل ما يمكن. يعد التكهن باستخدام الثبكات العصبية من الاساليب الحديثة التي لاقت اهتماما

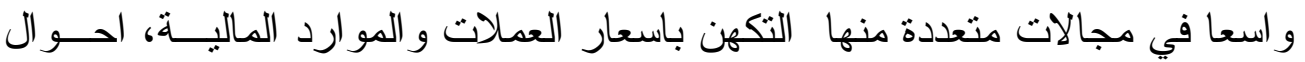
الطقس، تدفق المياه في الانهار، و استهلاكك الطاقة الكهربائية. و واســتخدمت بثـــــل

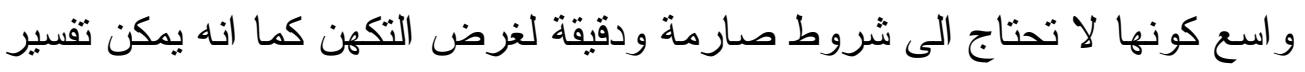
سلوك البيانات غير الخطي. ويمكن تلخيص عمل شبكة الانتشار العكسي للخطأ في التكهن بالخطوات الاتية (Sinha, 2002) :

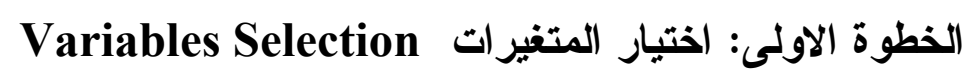
اذ يجب اختيار المشاهدات للمتغير ات بحيث تمنت المشكلة تمثيلاً جيداً.

Data Processing الخطوة الثانية: معالجة البيانات اجر اء بعض العمليات على البيانات المستخدمة، منل تحديد الاتجــاه العــام، النزكيز على العلاقات بين المشاهدات، ايجاد نوزيع البيانات. 
Divide data into Sets الخطوة الثالثة: تقسيم البيانات الى مجاميع تقسم البيانات المتوفرة الى المجاميع الاتية: مجموعة التدريب Training set: مجمو عة تعلم وتحديد نموذج للبيانات. مجمو عة الاختبار Testing set: و التي يمكن عن طريقها يتم تقدير مهارة الثبكة الافتز اضية و امكانية استخدامها بصورة عامة. مجموعة التحقيق Validation set: وهي مجموعة لاجر اء اختبار نهائي

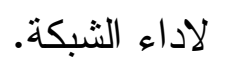

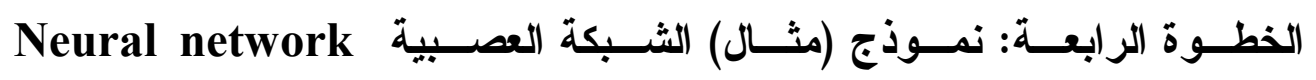
paradigms

عند تحديد نموذج الثبكة العصبية يجب اختيار: 廈 廈 廈 廈

Transfer function الخطوة الخامسة: دالة التحويل

الصيغة الرياضية لتحديد الاخر اج و التي تمنع الاخر اج من الوصول الـى قيمة عالية جدا وتستخدم احدى الصيخ او الدو ال الاتية : $\begin{array}{cr} & \text { Linear } \\ \text { 廈 } & \text { Threshold } \\ \text { 廈 } & \text { Sigmoid }\end{array}$

Evaluation Criteria الخطوة السادسة: معيار التقويم

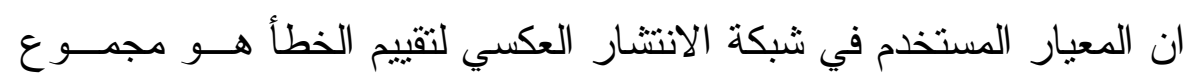
مربعات الاخطاء Mean Square Error (MSE). 


\section{Neural Network Training الخطوة السابعة: تدريب الثبكة} و وتتضمن هذه الخطوة:

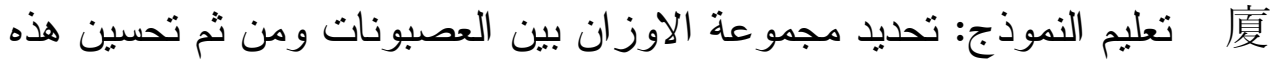
الاوزان نتيجة التدريب و التي تحدد اقل قيمة لمربع الخطأ للوصول الــى اوزان تعطي نتائج دقيقة. 廈

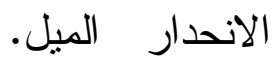

\section{الخطوة الثامنة: التنفيذ Implementation}

و هي من اهم الخطوات، اذ تختبر الثبكة من حيث قدرة التكيف مـــع حالـــة التغير في الدورة وامكانية اعادة التذريب و الوصول الى اقل مربع خطأ عند تغير البيانات الى الوصول الى حالة الاستقر ار. 4- التكهن باستهلاك الطاقة الكهربائية في مدينة الموصل لئل

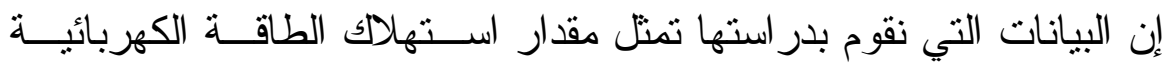

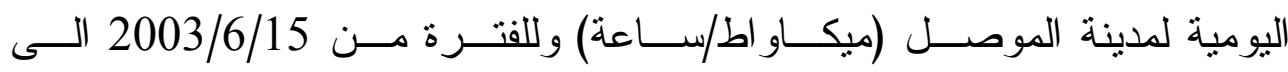

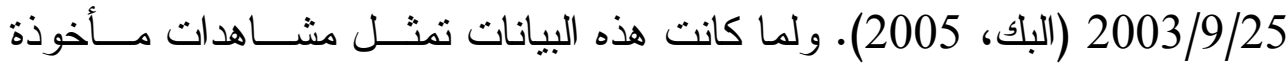
بازمنة مختلفة وان قيمها تتغير بتغير الزمن، فمن الو اضح التح انها تمثل بيانات لسلسلة زمنية ولتكن $\quad$... الكهربائية (ميكاو اط/ساعة) باليوم (t) في مدينة الموصل. وصف السلسلة الزمنية ان من اولى الخطوات التي تتبع لوصف او تحليل سلسلة زمنية هي رسمها

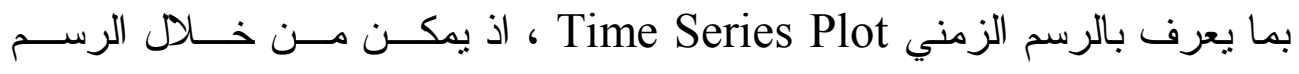

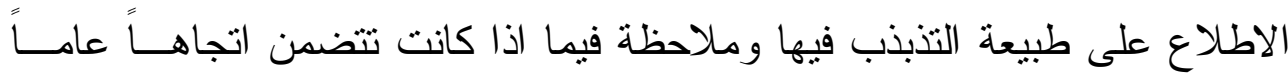
Trend الزمنية فضلاً عن علاقته الوثيقة بالتكهن. 
ييين الثكل (2) رسم السلسلة الزمنية التي نحسن بصــدها، ويلاحـــ ان

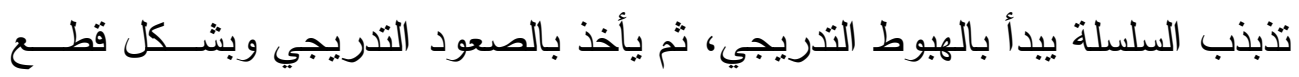

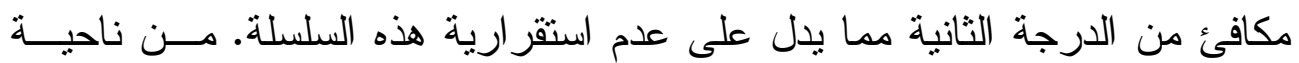

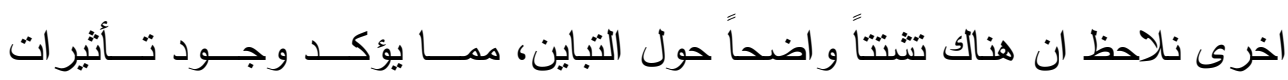
عشو ائية و اضحة ومؤثرة في هذه السلسلة.

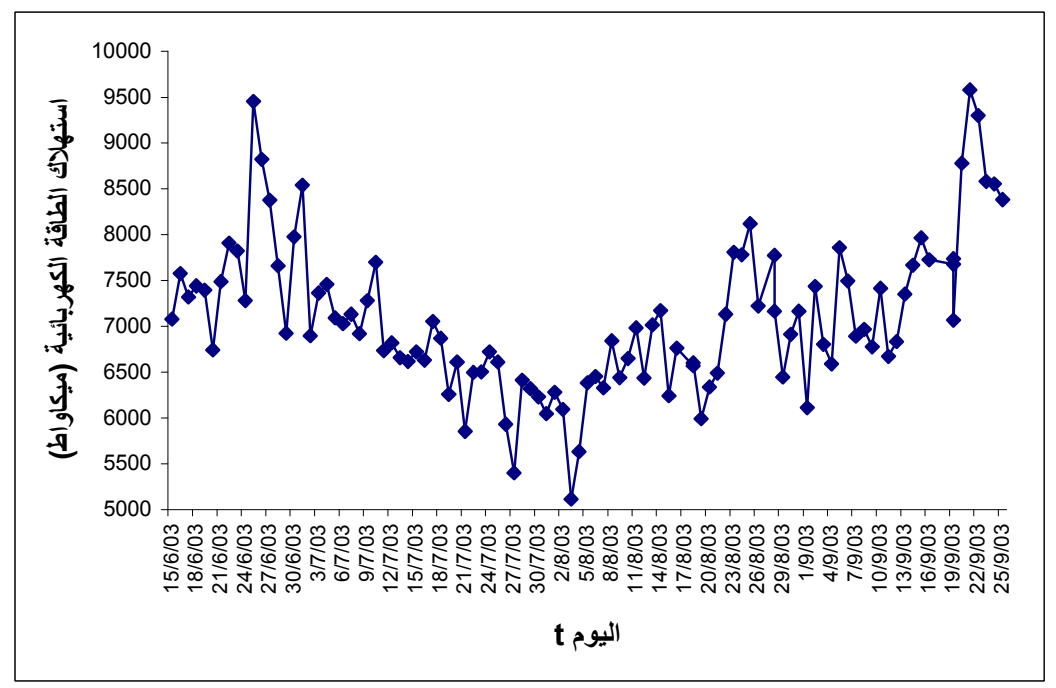

الثكل (2): الرسم الزمني لمشاهدات استهلاك الطاقة الكهربائية في مدينة الموصل.

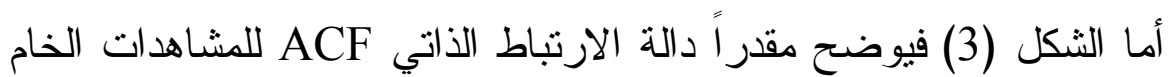

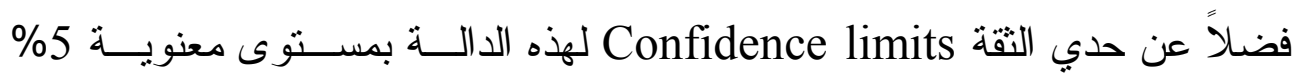
و الممنلة بالرسم بخطين افقيين منو ازيين ومنقطين.

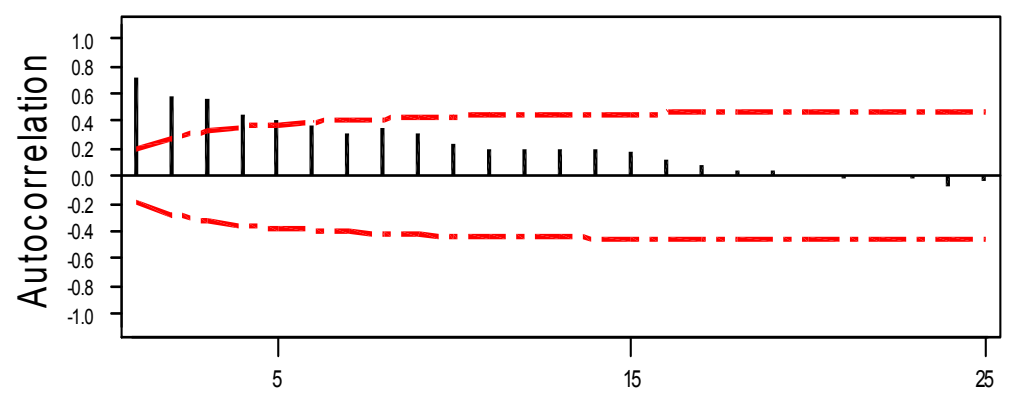

الثكل (3): دالة الارتباط الذاتي Autocorrelation للمشاهدات الخام. 
ولتحويل السلسلة الزمنية الى سلسلة مستقرة، اذ يشترط في التكهن بالطر ائق الكلاسيكية أن تكون السلسلة مستقرة. لقد تم إجر اء التحويل اللوغارتمي لمشــاهدات

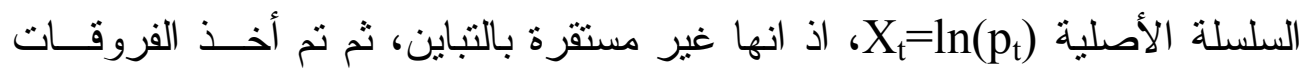
وبداً بالفرق الأول ( $)$ (Differences) الاتجاه العام تزبيعياً كما هو متوقع فقد اعطى الفرق الثــاني قريباً من التذبذب المستقر • بعد الحصول على المستقرة في سلوك السلسلة (الثكل 4) فقد تم العدـلـ مــن أجل تحديد النموذج المالائم ورتبته، وذللك من خلال حساب دالـــة الارتبـــاط الــذاتي

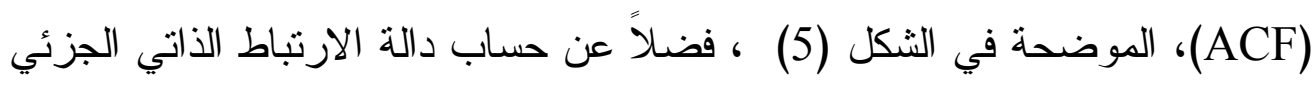
(PACF)

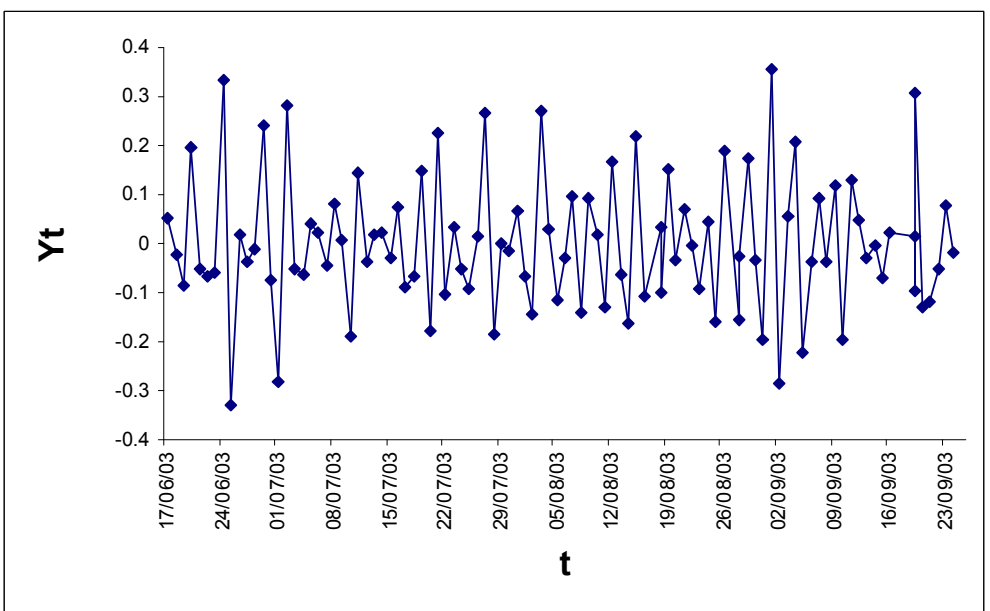

الثكل (4): سلوك المشاهدات بعد تحويل السلسلة الزمنية الى سلسلة مستقرة. 


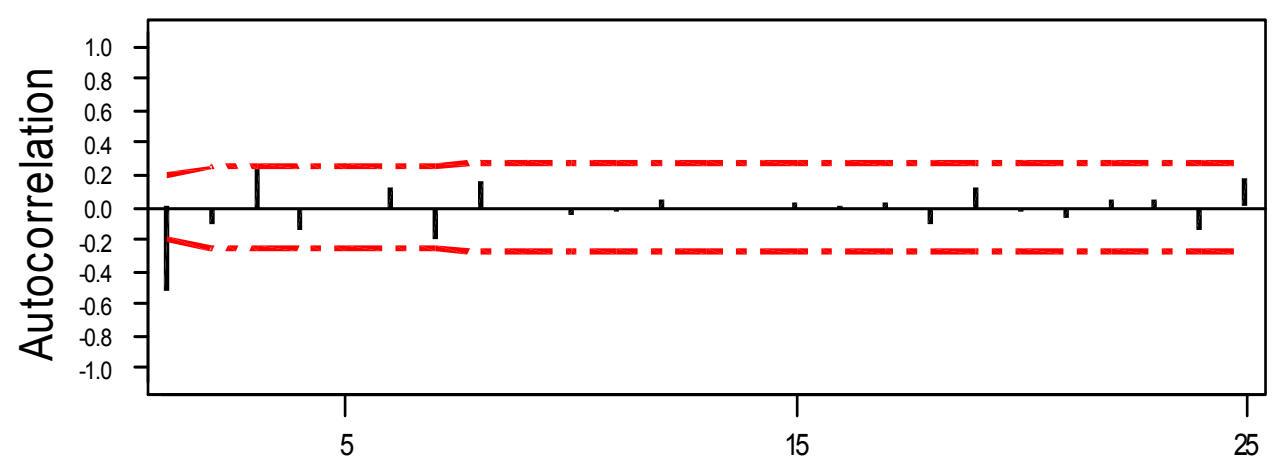

الثكل (5): دالة الارتباط الذاتي للسلسلة المستقرة $\}$

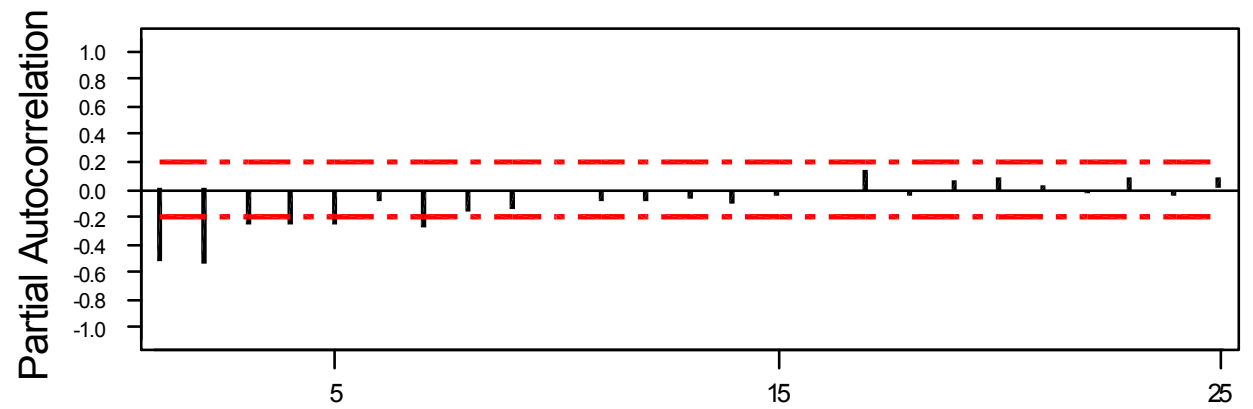

الشكل (6): دالة الارتباط الجزئي للسلسلة المستقرة $\left\{Y_{t}\right.$.

يتبين من الثكلين (5) و (6) ان النموذج المناســب للسلســلة نموذج ARMA(2,1) .وبعد أن تمت ملاعمة النموذج و التأكد من صلاحيته يــتم توظيفه الان لغرض التكهن. لو كتبنا النموذج الملائم بالثكل الاتي: $\mathrm{Y}_{\mathrm{t}}+\mathrm{a}_{1} \mathrm{Y}_{\mathrm{t}-1}+\mathrm{a}_{2} \mathrm{Y}_{\mathrm{t}-2}=\varepsilon_{\mathrm{t}}+\mathrm{b}_{1} \varepsilon_{\mathrm{t}-1}$

$$
\mathrm{Y}_{\mathrm{t}+1}=-\mathrm{a}_{1} \mathrm{Y}_{\mathrm{t}}-\mathrm{a}_{2} \mathrm{Y}_{\mathrm{t}-1}+\varepsilon_{\mathrm{t}+1}+\mathrm{b}_{1} \varepsilon_{\mathrm{t}}
$$

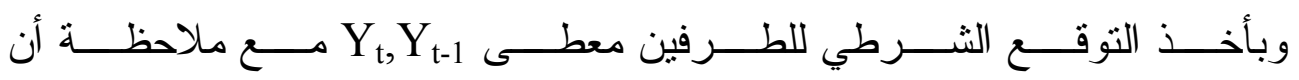
E( $\left(\varepsilon_{t+1} \mid Y_{t}, Y_{t-1}, \ldots\right)=0$

$\hat{\mathrm{Y}}_{\mathrm{t}+1}=-\mathrm{a}_{1} \mathrm{Y}_{\mathrm{t}}-\mathrm{a}_{2} \mathrm{Y}_{\mathrm{t}-1}+\mathrm{b}_{1} \varepsilon_{\mathrm{t}}$

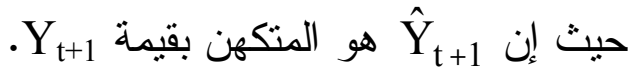

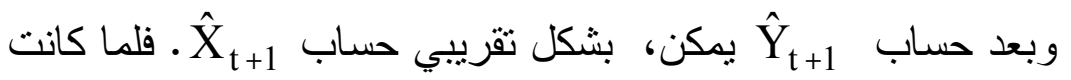
$Y_{t}=\Delta^{2} X_{t}=X_{t}-2 X_{t-1}+X_{t-2}$ 


$$
\hat{\mathrm{X}}_{\mathrm{t}+1}=\mathrm{Y}_{\mathrm{t}+1}+2 \mathrm{X}_{\mathrm{t}}-\mathrm{X}_{\mathrm{t}-1}
$$

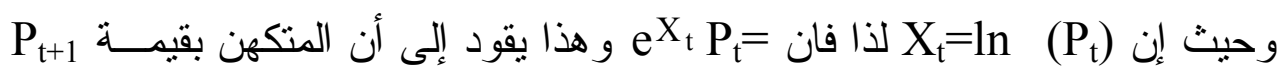

$$
\begin{aligned}
& \text {. } \hat{\mathrm{P}}_{\mathrm{t}+1}=\mathrm{e}^{\hat{\mathrm{X}}_{\mathrm{t}+1}} \quad \text { يوف وفون } \\
& \text { 5- استخدام الشبكات العصبية في التكهن }
\end{aligned}
$$

من الامور التي تشجع استخدام الثبكات العصبية فـي معالجـــة السلســلة

الزمنية التي نحن بصددها هو ان هذه السلسلة ليست ذات مو اصفات قياسية (كونها غير مستقرة)، كما ان عملية تحويلها باخذ الفروقات ليست محبذة دائما ما لم يكـن التوزيع الاحتمالي للسلسلة الاصلية تتوزع توزيعاً طبيعياً Normal. إن الثبكات العصبية يمكن استخدامها لمعالجة السلاسل الزمنية غير الخطية، فضلا عن انه من المفيد من الناحية العلمية الاطلاع على هذا الاسلوب الحديث ومقارنته مع الاساليب الكلاسيكية للتعرف على امكانياته وكفاءته في اجر اء التكهن.

لقد تم تصميم برنامج حاسوبي للحصــول علــى قـيم الــتكهن لسلســلة المشاهدات دون الحاجة الى معالجة عدم الاستقرارية، وان اول خطوة في استخدام البرنامج هي تحديد المدخلات للثبكة العصــبية، اذ ان المــدخلات هـــي الطاقــة الكهربائية المستهلكة، وتحديد عدد العقد المخفية و الذي يتحدد من خلال التـدريب و الذي يتضمن اجر اء العديد من التجارب الحاسوبية، وكذللك يمكن حسابه من خلال المعادلة التي قدمها (Lin et al., 1995) اذ ذكروا بان عدد العقد المخفبــة يمكــن

$$
\mathrm{N}_{\text {hidden }} \leq \frac{\mathrm{N}_{\text {train }} \mathrm{E}_{\text {tolerance }}}{\mathrm{N}_{\text {pts }}+\mathrm{N}_{\text {output }}}
$$

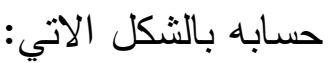

حيث ان: Nidden يمنل عدد العقد المخفية.

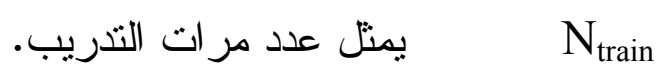

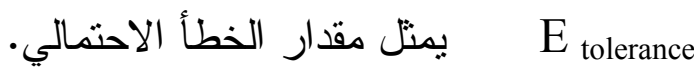
يمثل عدد البيانات التي تم اجر اء التدريب عليها. N

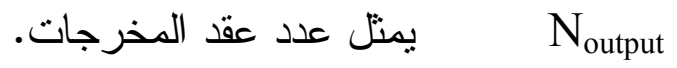


وفي هذه الدر اسة تم تثبيت مقدار الخطأ E tolerance كي يكون 0.01 و عدد مـر ات التدريب N $\mathrm{N}_{\text {train }}=500 \mathrm{XN}_{\mathrm{pts}} \mathrm{N}_{\text {output }}=1$ وبتطبيق المعادلة (2) نجد ان: $\mathrm{N}_{\text {hidden }} \leq \frac{5 \mathrm{~N}_{\text {pts }}}{\mathrm{N}_{\text {pts }}+1}$

لذا فان عدد العقد المخفية يجب ان يكون 4 للبيانات وحسب عدد مرات التذريب. وبما ان عدد البيانات المستخدمة في هذه الدراسة يبلغ (103) مشاهدات فقد اعتبــر (13\%) منها على انها مشاهدات اختبار Test لغرض الاختبار ، و اعتبر 15\% من

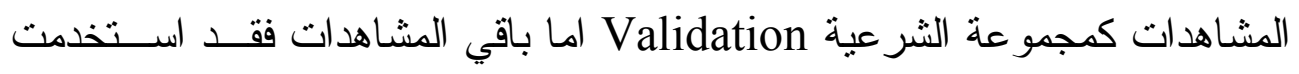
للتدريب وتقدير المعايير الاحصائية.

6- المقارنة بين الطريقة الاحصائية وطريقة الشبكات العصبية لغرض المقارنة بين الاسلوبين المستخدمين، وهما الاسلوب الكلاســيكي المتمثــلـ

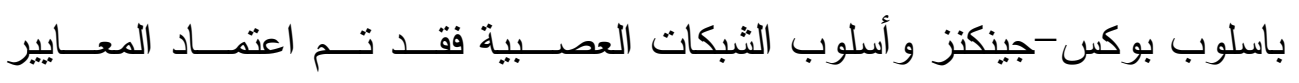
الاحصائية الآتية في المقارنة: معدل القيم المطلقة للاخطاء MAE. معدل مربعات الخطأ معثل

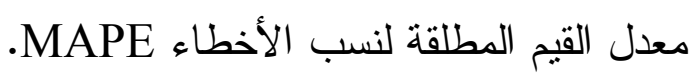
و الجدول الاتي يبين المعايير الاحصائية في المقارنة.

الجدول (1) :المعايير الاحصائية للتكهن باستهالاك الطاقة الكهربائية في مدينة

الموصل باستخدام الطر ائق الاحصائية و الشبكات العصبية.

\begin{tabular}{|c|c|c|r|}
\hline MAE & MSE & MAPE & بوكس -جينكنز الطريقة المستخدمة \\
\hline 780.584 & 1065722 & 11.243 & 3.367 \\
\hline 238.577 & 97978.9 & الثبكات العصبية \\
\hline
\end{tabular}

وكما هو و اضح فان اسلوب الثبكات العصبية متفوق على طريقة بوكسجينكنز ووفق المعايير الاحصائية الثلاثة. ويبين الثكل (7) قيم التكهن المحسوبة بالطريقتين المستخدمتني، ويتضح من الثكل ان منحنى الثبكة العصبية هو الافضل في قيم التكهن. 

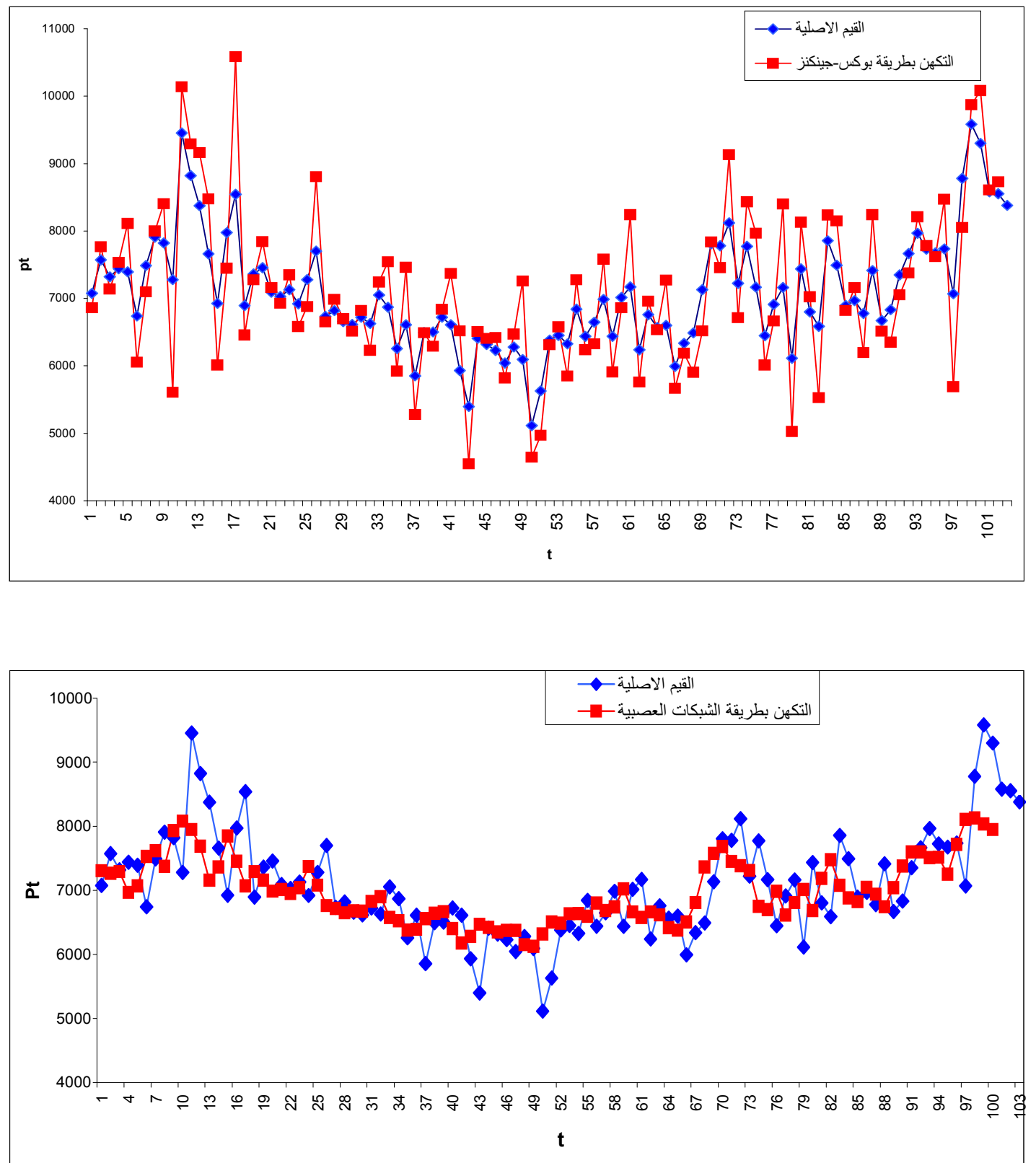

الثكل (7) قيم التكهن المحسوبة بالطريقتين الكلاسيكية و الثبكات العصبية. 
يستتتج من ذللك تفوق الثبكات العصبية على الطر ائق الكلاسيكية، اذ تم الحصــــل

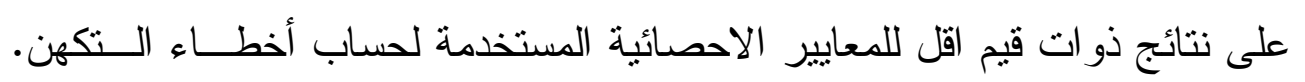
لذلك تعتبر الثبكات العصبية الاصطناعية هي الطريقة الأفضل و الأكثر دقة للنكهن

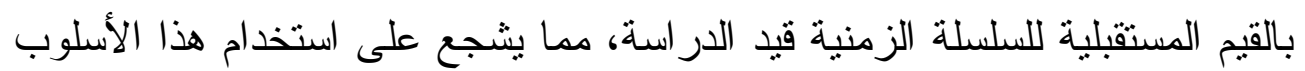

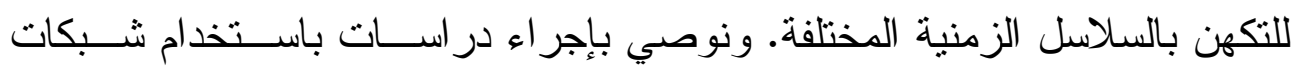

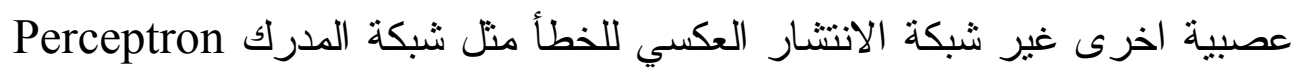
او الثبكة الدورية Recurrent Neural Network ومقارنة النتائج •

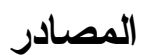

1. البك، عزة حازم .(2005)." استخدام الثبكات العصبية في الــكهن بالسلســلة

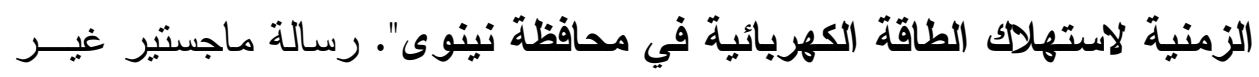

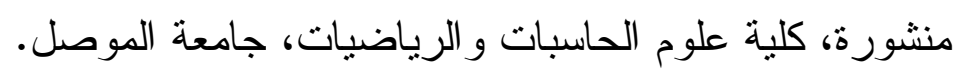

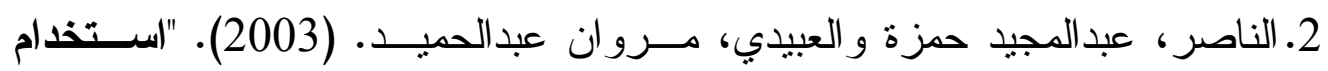

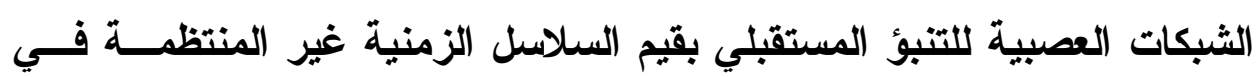
طول الموسمية"، المجلة العر اقية للعلوم الاحصائية، المجلد 3، العـدد 6، كليــة علوم الحاسبات و الرياضيات، جامعة الموصل.

3. Atiya , A. F.; El-Shoura, S. M.; Shaheen, S. I. and El-Sherif, M. S. (1999). “A Comparison Between Neural-Network Forecasting Techniques-Case Study: River Flow Forecasting", IEEE Transactions On Neural Networks, Vol. 10, No. 2, March .

4. Box,G.P. and Jenkins,G.M.(1976)."Time Series Analysis

Forecasting and Control", Revised Edition Holden-Day Inc. San Francisco.

5. Gershenson, Carlos. (1998). “Artificial Neural Networks for Beginners", Sussex Academy, UK. 
6. Lewis, P. A. W.; McKenzie, E. and Hugus, D. K. (1989). "Gamma Processes", Commune Statistic, Stochastic Model, 5(1), 1-30.

7. Lin, Feng; Yu, Xing Huo; Gregor, Shirely and Irons, Richard. (1995). "Time Series Forecasting with Neural Networks", Complexity International, Volume 02, ISSN 1320-0682, Australia.

8. Lotric, U. and Dobnikar ‘ A. (2001). "Wavelet Based Smoothing in Time Series Prediction with Neural Networks", University of Ljubljana, Faculty of Computer and Information Science, Slovenia.

9. Sansom , D. C. and Saha, T. K .Saha. (1999). "Neural Networks For Forecasting Electricity Pool Price In A Deregulated Electricity Supply Industry", Department of Computer Science and Electrical Engineering, University of Queensland.

10. Sinha, H. (2002). "Designing a Neural Network for forecasting Financial and Economic Time Series", June $4^{\text {th }}$, IND E 593.

11. Tong, Howell. (1990). "Non-linear Time Series, A Dynamical System Approach", Institue of Mathematics and Statistics, University of Kent at Conterbury, Clarendon Press Oxford.

12. Whittle, P. (1963). "Prediction and Regulation". English University Press, London, UK.

13. Zurada, Jacek M.and Cholewo ,Tomasz J.(1997). "Sequential Network construction for Time Series Prediction", In Proceedings of the IEEE International Joint Conference on Neural Networks, pp. 2034-2039, Houston, Texas, USA. 